\title{
SÍNDROME CARDIORRENAL EM CÃES: A BUSCA POR MEDIDAS DE CONTROLE
}

\author{
Marina Martins de Oliveira ${ }^{1}$ \\ Lorena Lorraine Alves Furtado ${ }^{1}$ \\ Claudine Botelho de Abreu ${ }^{1}$ \\ Tatiana Schulien ${ }^{2}$ \\ Ana Maria Barcelos Guerra Pinto ${ }^{3}$ \\ Luiz Eduardo Duarte de Oliveira ${ }^{1}$ \\ Mariana de Resende Coelho ${ }^{1}$ \\ Ruthnéa Aparecida Lázaro Muzzi ${ }^{1}$
}

\begin{abstract}
Resumo
A relação fisiopatológica entre os rins e o coração na doença, conhecida como síndrome cardiorrenal (SCR), envolve distúrbios do coração e dos rins, pois a disfunção aguda ou crônica em um órgão pode induzir a disfunção aguda ou crônica do outro. Em Medicina Veterinária são descritos 5 subtipos de síndrome cardiorrenal: cardiorrenal aguda, cardiorrenal crônica, renocárdica aguda, renocárdica crônica e secundária. A anemia é um achado comum em cães com disfunção cardíaca e renal, caracterizando a chamada síndrome da anemia cardiorrenal. Os mecanismos envolvidos na fisiopatologia da síndrome cardiorrenal envolvem, não somente alterações hemodinâmicas e ativação de sistemas neuro-hormonais, como também a ativação de mecanismos compensatórios do próprio organismo, que acabam contribuindo para a piora da função cardíaca e renal, levando ao desenvolvimento da SCR. O grande desafio encontrado em medicina veterinária está relacionado ao diagnóstico precoce dessa síndrome e, principalmente, ao correto manejo terapêutico, uma vez que que a terapia da cardiopatia pode deteriorar a função renal, e vice-versa. Portanto, é importante compreender como a fisiopatologia de uma doença pode impactar a função do outro órgão. O presente trabalho tem como objetivo trazer uma completa abordagem da fisiopatologia, diagnóstico e tratamento da síndrome cardiorrenal em cães.
\end{abstract}

Palavras-chave: cardiopatia, cães, nefropatia, anemia.

\section{CARDIORENAL SYDROME IN DOGS: THE INVESTIGATION FOR CONTROL MEASURES}

\begin{abstract}
The physiopathological relationship between the kidneys and the heart in the disease, known as cardiorenal syndrome (CRS), involves disorders of the heart and kidneys, since acute or chronic dysfunction in one organ can induce acute or chronic dysfunction of the other. In veterinary medicine, 5 subtypes of cardiorenal syndrome are described: acute cardiorenal, chronic cardiorenal, acute renocardial, chronic and secondary renocardial. Anemia is a common finding in dogs with cardiac and renal dysfunction, characterizing the so-called cardiorenal anemia syndrome. The mechanisms involved in the pathophysiology of the cardiorenal syndrome involve not only hemodynamic changes and activation of neurohormonal systems, but also the activation of compensatory mechanisms of the organism itself, which end up

\footnotetext{
${ }^{1}$ Universidade Federal de Lavras, Lavras, MG, Brasil. claudinebabreu@ hotmail.com

${ }^{2}$ Universidade Vale do Rio Verde, Três Corações, MG, Brasil

${ }^{3}$ University of Lincoln, Lincoln, Lincolnshire, UK, Inglaterra.
} 
contributing to worsening cardiac and renal function, leading to the development of CRS. The great challenge found in Veterinary Medicine is related to the early diagnosis of this syndrome and, mainly, to the correct therapeutic management, since that the therapy of the cardiopathy can deteriorate the renal function, and vice versa. Therefore, it is important to understand how the pathophysiology of one disease can impact the function of the other organ. The present work aims to bring a complete approach to the pathophysiology, diagnosis and treatment of cardiorenal syndrome in dogs.

Keywords: cardiopathy, dogs, nephropathy, anemia.

\section{SÍNDROME CARDIORRENAL EN PERROS: LA BÚSQUEDA DE MEDIDAS DE CONTROL}

\section{Resumen}

La relación fisiopatológica entre los riñones y el corazón en la enfermedad, conocida como síndrome cardiorrenal (SCR), involucra trastornos cardíacos y renales, ya que la disfunción aguda o crónica en un órgano puede inducir disfunción aguda o crónica en el otro. En medicina veterinaria se describen 5 subtipos de síndrome cardiorrenal: cardiorrenal agudo, cardiorrenal crónico, renocardial agudo, renocardial crónico y secundario. La anemia es un hallazgo común en perros con disfunción cardíaca y renal, que caracteriza el llamado síndrome de anemia cardiorrenal. Los mecanismos involucrados en la fisiopatología del síndrome cardiorrenal implican no solo cambios hemodinámicos y activación de los sistemas neurohormonales, sino también la activación de mecanismos compensatorios del propio cuerpo, que en última instancia contribuyen al empeoramiento de la función cardíaca y renal, lo que conduce al desarrollo de CRS. El principal desafío que se encuentra en la medicina veterinaria está relacionado con el diagnóstico precoz de este síndrome y, especialmente, con el tratamiento terapéutico correcto, ya que la terapia de la enfermedad cardíaca puede deteriorar la función renal y viceversa. Por lo tanto, es importante comprender cómo la fisiopatología de una enfermedad puede afectar la función del otro órgano. El presente trabajo tiene como objetivo brindar un enfoque completo de la fisiopatología, el diagnóstico y el tratamiento del síndrome cardiorrenal en perros.

Palabras clave: enfermedad cardíaca, perros, nefropatía, anemia.

\section{INTRODUÇÃO}

A síndrome cardiorrenal é definida como a interação entre coração e rim, em que o comprometimento de um órgão está associado ao comprometimento do outro por distúrbios agudos ou crônicos. A interação entre esses dois sistemas envolve diversos mecanismos relacionados à regulação de volume e à disfunção (1-3). De acordo com o que foi proposto pelo Grupo de Consenso em Síndrome Cardiorrenal, os distúrbios do eixo cardiovascular-Renal são induzidos pela doença, por toxinas, drogas, por danos estruturais e/ou funcionais ao rim ou ao sistema cardiovascular, levando a uma interrupção das interações normais entre esses sistemas (2). As desordens cardiovasculares são secundárias à diminuição do débito cardíaco e causam diminuição da perfusão renal, podendo levar ao aumento da ureia e da creatinina séricas, ativação do sistema-renina-angiotensina-aldosterona-ADH (SRAAA) e redução da taxa de filtração glomerular, gerando menor produção urinária (4). A maioria das lesões renais gera efeitos cardiovasculares, como hipertensão sistêmica, hipertrofia de ventrículo esquerdo, desregulação do volume do fluido e complicações de doenças subjacentes (5). 
Na literatura são descritos cinco subtipos de síndrome cardiorrenal: tipo 1 (síndrome cardiorrenal aguda), quando uma disfunção cardíaca aguda leva à lesão também aguda nos rins; tipo 2 (síndrome cardiorrenal crônica), quando anormalidades cardíacas crônicas levam à doença renal crônica que está progredindo; tipo 3 (síndrome renocárdica aguda), quando a piora súbita da função renal leva a disfunção cardíaca aguda; tipo 4 (síndrome renocárdica crônica), quando a doença renal crônica primária contribui para o início da disfunção cardíaca, hipertrofiando ventrículos, causando disfunção diastólica, aumentando de risco de eventos cardiovasculares adversos ou uma combinação desses fatores; e tipo 5 (síndrome cardiorrenal secundária), quando a combinação da doença cardíaca e renal está associada a distúrbios sistêmicos agudos ou crônicos, como sepse, levando à piora do quadro clínico $(1-4,6,7)$.

A doença valvar mitral crônica relaciona-se com o aumento da prevalência de doenças renais. A doença cardíaca grave, doença renal grave, administração de diuréticos, idade avançada e o diagnóstico clínico da síndrome cardiorrenal foram associados ao menor tempo de sobrevida. O tratamento para a doença cardíaca pode levar ao desenvolvimento de uma doença renal nos pacientes e as síndromes cardiorrenais pioram o prognóstico (4). A disfunção cardíaca secundária à lesão renal aguda pode ocorrer pela ativação do sistema imunológico, que gera efeitos diretos no coração, e pela sobrecarga de volume sanguíneo, a hipertensão e a acidose, que geram efeitos indiretos na função cardíaca (1).

\section{A ANEMIA ASSOCIADA À SÍNDROME}

$\mathrm{Na}$ medicina, a anemia está frequentemente associada à insuficiência cardíaca ou renal (2). Na Medicina Veterinária a presença de anemia, associada à síndrome cardiorrenal, forma uma tríade descrita na literatura como síndrome da anemia cardiorrenal $(8,9)$. Ela ocorre em $8,1-22,8 \%$ dos cães (4).

Em um estudo realizado por Martinelli et al. (4) para avaliar a anemia como fator agravante da doença valvar crônica de mitral foi observada uma prevalência de $15 \%$ de anemia, mas não houve diferenças entre a prevalência de anemia em diferentes classes de doença cardíaca e nenhuma diferença entre os grupos de animais tratados e não tratados para insuficiência cardíaca. Nesse mesmo estudo, a prevalência de anemia foi significativamente maior no grupo de animais com azotemia do que no grupo sem azotemia e maior na fase de doença renal crônica (DRC) avançada. Portanto, a hemoglobina foi correlacionada negativamente com o valor da creatinina sérica e o estágio da DRC, confirmando a existência de uma ligação entre o grau de insuficiência renal e a anemia.

\section{A ATIVAÇÃO DO SISTEMA RENINA-ANGIOTENSINA-ALDOSTERONA-ADH (SRAAA)}

A redução do débito cardíaco associada a uma consequente redistribuição central do fluxo sanguíneo resulta em hipoperfusão renal. Então, mecanismos compensatórios, como o SRAAA, aumentam a reabsorção de sódio e água visando aumentar a pressão arterial e restabelecer a homeostase (4). Além disso, existe um mecanismo de feedback negativo para a liberação de renina por meio da ação intra-renal direta da angiotensina II (6). Esses efeitos, em longo prazo, têm a capacidade de promover fibrose em cardiomiócitos e remodelar os ventrículos. Por outro lado, a hipoperfusão gera uma isquemia renal, tanto no parênquima quanto no córtex, que também compromete a função desse órgão (10). O SRAAA medeia reações oxidativas dependentes de citocinas, inflamação e morte celular. Isso gera a formação de espécies reativas a oxigênio (EROs), que fornecem uma ligação importante entre as disfunções renais e cardíacas, levando também a deficiência no transporte de cálcio e outros íons. O estresse oxidativo aumenta a apoptose de células renais e cardíacas (11). 
A manutenção do sódio em valores normais é o tratamento preconizado, tanto para a hipertensão, quanto para a síndrome cardiorrenal. O bloqueio do SRAAA mostrou benefícios em pacientes humanos com resistência à insulina e obesidade. A ativação desse sistema leva a uma diminuição do óxido nítrico circulante, diminuindo sua biodisponibilidade tecidual e levando à vasoconstrição. Em humanos, a produção de angiotensinogênio pelos adipócitos pode representar até 30\% da substância circulante, além de um aumento do sistema nervoso simpático, provavelmente pelo hormônio leptina, que reduz a natriurese e diminui o óxido nítrico. O tecido adiposo é responsável por parte da formação do angiotensinogênio e aldosterona extra-renal, contribuindo para a ativação do SRAAA (12).

\section{O DIAGNÓSTICO E A TERAPIA}

A manifestação da doença cardíaca pode incluir anormalidades funcionais, arritmias e concentrações elevadas de troponina cardíaca no soro sanguíneo. A concentração sérica da troponina cardíaca I é considerada a análise sanguínea mais sensível para danos no miocárdio. Estudos demostram que outras doenças como insuficiência renal, leptospirose (5) e doenças que podem levar a sepse, como a piometra $(1,13,14)$, têm probabilidade de induzir lesões miocárdicas e aumentar a concentração de troponina I. Esses autores levantaram a hipótese de que a insuficiência renal aguda está associada a arritmias cardíacas e avaliaram a relação das lesões funcionais e estruturais em cães com doença renal. Concluíram que arritmias e lesões miocárdicas acontecem em cães com doença renal e a terapia de reposição pode aumentar o risco de hipotensão, hipovolemia, mudança no padrão de eletrólitos e fluidos, causando lesão miocárdica (5).

Atualmente não existem biomarcadores específicos para a síndrome cardiorrenal, sendo a parte renal sempre avaliada pela função ou dano renal, e a cardíaca pelo peptídeo natriurético atrial e troponina I (4). A ultrassonografia abdominal permite avaliar anormalidades no fluxo sanguíneo, auxiliando no diagnóstico da doença renal (5). As concentrações de glicose e aminoácidos na urina são usadas para avaliar a função renal tubular proximal, enquanto as concentrações de eletrólitos e bicarbonato refletem a capacidade do rim de manter o equilíbrio hidroeletrolítico e acidobásico. A dificuldade do diagnóstico pode levar a subestimação da síndrome cardiorrenal nos pacientes (4).

Alguns pesquisadores notaram uma prevalência da doença renal crônica complicando a doença cardíaca em cães (4). Tanto o coração quanto o rim são afetados pelo volume anormal dentro dos vasos, pela pressão arterial sistêmica descontrolada e pelos tratamentos comumente empregados: diuréticos, vasodilatadores, fluidos suplementares (2). O estágio da doença renal também influencia a condição cardíaca e, quanto pior essa, maior a dose de diurético necessária, criando um ciclo (4). A insuficiência cardíaca é a condição em que o coração doente não fornece um débito cardíaco adequado ou só consegue fazê-lo na presença de pressões arteriais elevadas. O tratamento de cardiopatias pode incluir o uso de diuréticos (furosemida e hidroclorotiazida), vasodilatadores (amlodipina, diltiazem, nitroglicerina, hidralazina e nitroprussiato de sódio), agentes bloqueadores neuro-hormonais (espironolactona), bloqueadores beta-adrenérgicos (atenolol e propranolol) e inotrópicos positivos (digoxina, dobutamina e pimobendan). O alvo do tratamento é a manutenção do volume e da pressão intravascular, permitindo uma perfusão renal suficiente e evitando a sobrecarga e desequilíbrio de fluidos, ao contrário do objetivo do tratamento da insuficiência cardíaca congestiva, que é reduzir o volume intravascular. O uso de diuréticos associado aos inibidores da enzima conversora de angiotensina aumenta o risco de lesão renal (2). A medida que piora o quadro renal, mais grave é a anemia dos cães avaliados (4).

A doença cardíaca mais comum em cães é a degeneração mixomatosa da valva mitral. Em um estudo realizado por Martinelli et al. (4), a prevalência de cães com doença cardíaca 
que apresentavam diminuição na função renal foi de $32 \%$ e houve correlação direta do remodelamento cardíaco com a piora da função renal. A ocorrência de azotemia em animais com degeneração de valvas cardíacas é comum, e aumenta as chances de haver falência cardíaca (15). Considerando que a utilização de diuréticos é necessária para tratamento de cães com degeneração mixomatosa da valva mitral, há recomendações de baixas doses desses fármacos, visando manter a função renal preservada, uma vez que o tratamento da disfunção cardíaca pode afetar os rins (4).

O atual aumento da expectativa de vida dos cães aumenta também os casos de síndrome cardiorrenal, devido à trivialidade das alterações cardíacas nesses animais. Isso se deve, principalmente, à utilização de diuréticos e inibidores da enzima conversora de angiotensina para tratamento das cardiopatias. Assim sendo, os diuréticos de alça devem ser utilizados na dose mínima suficiente para aliviar os sintomas do edema. Deve-se atentar ao uso de outros fármacos como digoxina, enalapril e atenolol, que podem causar toxicidade em casos de nefropatias (como arritmias, hipotensão e piora da função miocárdica), uma vez que possuem excreção renal (12). O volume de fluido e o estado hemodinâmico são quase sempre anormais em pacientes renais e a lesão renal também pode levar a sobrecarga de volume sistêmico, contribuindo para o congestionamento, especialmente em animais com doença valvar, cardiomiopatia dilatada, disfunção diastólica e anemia. Ademais, a própria azotemia pode gerar efeitos maléficos nos cardiomiócitos (4).

A ocorrência da síndrome cardiorrenal torna o prognóstico desfavorável (1) devido ao ciclo formado pela disfunção de ambos os órgãos (4) e a dificuldade de ajuste à terapia (2).

\section{CONSIDERAÇÕES FINAIS}

A síndrome cardiorrenal é uma doença comum em cães, principalmente naqueles que possuem um acometimento cardíaco prévio. O diagnóstico desta síndrome não possui um marcador específico, portanto são necessárias pesquisas nessa área para estabelecer um método diagnóstico mais preciso e precoce, visando a existência de tempo hábil para o médico veterinário instituir uma terapia cardíaca adequada, atentando-se aos possíveis danos renais. A terapêutica cardíaca afeta os rins, seja devido ao uso excessivo de diuréticos ou pela metabolização de outros fármacos. Em contrapartida, a fluidoterapia, que é uma das formas preconizadas para restabelecer a função renal, pode prejudicar o animal cardiopata. Isso causa episódios de desestabilização da doença, o que pode envolver hipertensão e edema pulmonar agudo, gerando um grande risco à vida do paciente. O ideal é que seja encontrada a menor dose efetiva para o tratamento da cardiopatia, de modo que a progressão do acometimento renal possa não ocorrer ou ocorrer de forma lenta, possibilitando ao profissional prover bem-estar a esse animal por um período prolongado.

\section{REFERÊNCIAS}

1. Athwani V, Bhargava M, Chanchlani R, Mehta AJ. Incidence and outcome of acute cardiorenal syndrome in hospitalized children. Indian J Pediatr. 2017;84(6):420-4. doi: 10.1007/s12098-017-2307-3.

2. Pouchelon JL, Atkins CE, Bussadori C, Oyama MA, Valden SL, Bonagura JD, et al. Cardiovascular-renal axis disorders in the domestic dog and cat: a veterinary consensus statement. J Small Anim Pract. 2015;56(9):537-52. doi: 10.1111/jsap.12387.

3. Smyth A, Ronco C, Garovic VD. Preeclampsia: a cardiorrenal syndrome in pregnancy. Curr Hypertens Rep. 2017;19(2):15. doi: 10.1007/s11906-017-0714-5. 
4. Martinelli E, Locatelli C, Bassis S, Crosara S, Paltrinieri S, Scarpa P, et al. Preliminary investigation of cardiovascular-renal disorders in dogs with chronic mitral valve disease. J Vet Intern Med. 2016;30(5):1612-8. doi: 10.1111/jvim.14524.

5. Keller SP, Kovacevic A, Howard J, Schweighauser A, Francey T. Evidence of cardiac injury and arrhythmias in dogs with acute kidney injury. J Small Anim Pract. 2016;57(8):4028. doi: 10.1111/jsap.12495.

6. Homma T, Sonoda H, Manabe K, Arai K, Mizuno M, Sada T, et al. Activation of renal angiotensin type 1 receptor contributes to the pathogenesis of progressive renal injury in a rat model of chronic cardiorenal syndrome. Am J Physiol Renal Physiol. 2012;302(6):50-67. doi: 10.1152/ajprenal.00494.2011.

7. Núñes J, Miñana G, Santas E, Bertomeu-González V. Cardiorenal syndrome in acute heart failure: revisiting paradigms. Rev Esp Cardiol. 2015;68(5):426-35. doi: 10.1016/j.rec.2014.10.016.

8. Nicolle AP, Chetboul V, Allerheiligen T, Pouchelon JL, Gouni V, Tessier-Vetzel D, et al. Azotemia and glomerular filtration rate in dogs with chronic valvular disease. J Vet Intern Med. 2007;21:943-9. doi: 10.1111/j.1939-1676.2007.tb03047.x

9. Ronco C, Maisel A. Volume overload and cardiorenal syndromes. Congest Heart Fail. 2010;16(4):1-4. doi: 10.1111/j.1751-7133.2010.00176.x.

10. Lekawanvijit S, Kompa AR, Manabe M, Wang BH, Langham RG, Nishijima F, et al. Cronic kdney disease-induced cardiac fibrosis is ameliorated by reducing circulating levels of a non-dialysable uremic toxin, indoxyl sulfate. PLoS One. 2012;7(7):1-10. doi: 10.1371/journal.pone.0041281.

11. Virzi GM, Clemente A, Cal M, Brocca A, Day S, Pastori S, et al. Oxidative stress: dual pathway induction in cardiorrenal syndrome type 1 pathogenesis. Oxid Med Cell Longev. 2015;2015:1-9. doi: 10.1155/2015/391790.

12. Cabandugama PK, Gardner MJ, Sowers JR. The renin angiotensin aldosterone system in obesity and hypertension: roles in the cardiorenal metabolic syndrome. Med Clin North Am. 2017;101(1):129-137. doi: 10.1016/j.mcna.2016.08.009.

13. Sims CR, Singh SP, Mu S, Gokden N, Zakaria D, Nguyen TC, et al. Rolipram improves outcome in a rat modelo of infant sepsis-induced cardiorenal syndrome. Front Pharmacol. 2017;8(237):1-4. doi: 10.3389/fphar.2017.00237.

14. Pereira CS, Muzzi RAL, Figueiredo VC, Oberlender G, Lacreta Júnior ACC, et al. Troponin I as a biomarker of cardiac injury in dogs with sepsis. Arq Bras Med Vet Zootec. 2016;68(4):919-26. doi: 10.1590/1678-4162-8696.

15. Yu IBY, Huang HP. Prevalence and prognosis of anemia in dogs with degenerative mitral valve disease. Biomed Res Int. 2016;2016:1-5. doi: 10.1155/2016/4727054. 
Recebido em: 05/09/2019

Aceito em: 25/11/2019 